\title{
Studies on the PMR Spectra of Oxetanes
}

\author{
IV. 2-(4-Halophenyl) oxetanes \\ JUKKA JOKISAARI and ERKKI RAHKAMAA \\ Laboratory of Molecular Spectroscopy, Department of Physics, University of Oulu \\ and Heikki Malo \\ Department of Chemistry, University of Oulu, Oulu, Finland \\ (Z. Naturforsch. 26 a, 973-978 [1971] ; received 28 February 1971)
}

\begin{abstract}
The $60 \mathrm{MHz}$ PMR spectra of 2-(4-iodophenyl) oxetane, 2-(4-bromophenyl) oxetane, 2-(4-fluorophenyl) oxetane, and 2-(4-chlorophenyl) oxetane have been analysed for the oxetane ring protons and the phenyl protons. In the three first mentioned compounds the spectra show a coupling of $0.46-0.63 \mathrm{~Hz}$ between the benzyl proton and the phenyl protons in ortho position relative to the oxetane ring, the other proton-proton couplings between the oxetane and phenyl parts being zero in the error limits.

In the oxetane part, the halogen substituent in the para position of the phenyl ring mainly affects the chemical shifts of the protons with a slight effect on the proton-proton couplings.

The ortho and meta couplings of the phenyl protons increase and the para coupling decreases with growing electronegativity of the substituent, in the order iodine, bromine, fluorine. The phenyl protons in 2-(4-chlorophenyl) oxetane produce a sharp singlet, which shows that they are chemically equivalent. This proves that chlorine and the oxetane group affect the shieldings of the phenyl protons similarly, which is supported by comparing the chemical shift differences in 2-(4-halophenyl) oxetanes to the ones in para-dihalo-substituted benzenes.
\end{abstract}

\section{Introduction}

The PMR investigations of oxetanes (trimethylene oxides) are relatively few. Primas, FreI, and GÜNTHARD $^{1}$ and LIPPERT and PRIGGE ${ }^{2}$ have reported the chemical shift values of oxetane protons. FERRETTI ${ }^{3}$, and LOZACH and BRAILLON ${ }^{4}$ have analyzed the PMR spectrum of oxetane. The values of the vicinal and geminal proton-proton couplings of 2,2- $\mathrm{d}_{2}$-oxetane have been obtained in the work of Lustig and collaborators ${ }^{5}$. YATES and SzaBo ${ }^{6}$ noted the values of the chemical shifts and geminal couplings for 3-substituted-oxetan-3-ols. SAmitov and co-workers ${ }^{7}$ have considered the configurations and conformations of 2-methyloxetane, 2,3-dimethyloxetane and 2-methyl-3-isobutyloxetane using $100 \mathrm{MHz}$ PMR spectra.

1 H. Primas, K. Frei, and Hs. H. Günthard, Helv. Chim. Acta 41, 35 [1958].

2 E. Lippert and H. Prigge, Ber. Bunsenges. 67, 415 [1963].

3 J. A. Ferretti, Ph. D. Thesis, Diss. Abstr. 26, 7060 [1966].

4 R. Lozach and B. Braillon, Bull. Soc. Chim. France 1967, 748; J. Chim. Phys. Physicochim. Biol. 67, 340 [1970].

5 E. Lustig, E. P. Ragelis, and N. Duy, Spectrochim. Acta 23 A, 133 [1967].

6 P. YAtes and A. G. Szabo, Tetrahedron Lett. 1965, 485.

7 Yu. Yu. Samitov, A. V. BogatskiI, and G. A. Filip, Dokl. Akad. Nauk SSSR 192, 138 [1970].

8 A. Danti, W. J. Lafferty, and R. C. Lord, J. Chem. Phys. 33, 294 [1960].
The complete analysis of the PMR spectrum of planar ${ }^{8-12}$ oxetane itself is possible only with very high resolution. Owing to the anisotropy of the magnetic susceptibility and probably also to the structural changes of the oxetane ring, produced by substitution, the 2 -substituted oxetanes produce more numerous resolved lines than oxetane itself in the PMR spectra recorded with the usual resolution of $0.3-0.4 \mathrm{~Hz}$, thus making a complete spectral analysis possible.

In the previous parts of this investigation the $60 \mathrm{MHz}$ PMR spectra of 2-phenyloxetane ${ }^{13}, 2$ - (4methylphenyl) -oxetane, 2-(2,4-dimethylphenyl) oxetane, 2-(2,4,6-trimethylphenyl) oxetane ${ }^{14}$, and 2 methyloxetane ${ }^{15}$ have been analyzed determining all the chemical shifts and proton-proton couplings

9 S. I. Chan, J. Zinn, and W. D. Gwinn, J. Chem. Phys. 33, 295 [1960].

10 S. I. Chan, J. Zinn, J .Fernandez, and W. D. Gwinn, J. Chem. Phys. 33, 1643 [1960].

11 S. I. Chan, J. Zinn, and W. D. Gwinn, J. Chem. Phys. 34, 1319 [1961].

12 S. I. Chan, T. R. Borgers, J. W. Russell, H. L. Strauss, and W. D. GWINN, J. Chem. Phys. 44, 1103 [1966].

13 J. Jokisaari, E. RahkamaA, and P. O. I. Virtanen, Suomen Kemistilehti B 43, 14 [1970].

14 J. JokisaAri and H. Ruotsalainen, Z. Naturforsch. 25 a, 1655 [1970].

15 J. JokisaArI, Phil. lic. thesis, University of Oulu, Oulu 1970. 
in the oxetane parts. The present work contains the analyses of the $60 \mathrm{MHz}$ spectra of the oxetane and phenyl protons in 2-(4-halophenyl) oxetanes.

\section{Experimental Methods}

\section{Materials}

The oxetanes were synthetized by the method of SEARLES et al. ${ }^{16}$ starting from halobenzenes and 3chloropropionyl chloride. The ketones were reduced with lithium aluminium hydride to alcohols, which were acetylated with acetyl chloride. The acetates were treated with hot alkali, giving substituted phenyloxetanes. All ketones and alcohols were purified by crystallization or distillation. The acetates and oxetanes were purified by distillation. The boiling points $\left({ }^{\circ} \mathrm{C} / \mathrm{mm} \mathrm{Hg}\right)$ and refractive indexes $\left(n_{\mathrm{D}}^{20}\right)$ of the used compounds ${ }^{17}$ were as follows: 2-(4-iodophenyl)-oxetane (I) 115/0.15, 1.6188; 2-(4-bromophenyl)oxetane (II) $111-112 / 0.40$, $1.5730 ; 2$-(4-fluorophenyl) oxetane (III) $99.5-100 / 17$, 1.5088; 2-(4-chlorophenyl)oxetane (IV) 89/0.15, 1.5477.

The PMR spectra show traces of unidentified impurity, the resonances, however, having no disturbing effect on the spectral analysis. All the compounds were dissolved into 10 mole per cent carbon tetrachloride solutions, and a small amount of TMS was added for internal reference.

\section{Recording of Spectra}

The spectra of the four oxetane derivatives were recorded at room temperature on a Varian A-60 spectrometer four times in both sweep directions. The spectra were calibrated by producing the TMS sidebands with a Krohn-Hite 4100 push-button oscillator. The values of calibration frequencies were determined as averages of several successive readings of an Advance TC 9 frequency counter.

The experimental line frequencies used for calculating the chemical shifts and couplings by iterative process were taken as averages of six best measurements. The inaccuracy of the experimental line frequencies, caused on one hand by the inaccuracy of the frequency counter, and on the other by the uncertainty in the measurements, can be regarded as $\pm 0.10 \mathrm{~Hz}$ or better.

\section{Computer Programs}

The theoretical spectra were calculated using programs ABCDEIT ${ }^{18}$, SIX ${ }^{15}$, and LAOCN $3{ }^{19}$. The shape of the triplet of the benzyl proton was calculated by the program MUOTO, which uses the Lorentzian line shape function and which has been written in this laboratory. Computations were performed on IBM $360 / 30$ and $/ 50$ systems.

16 S. Searles, Jr., K. A. Pollart, and E. F. Lutz, J. Amer. Chem. Soc. 79, 948 [1957].

17 P. O. I. Virtanen, H. Malo, and H. Ruotsalainen, Suomen Kemistilehti B 43, 512 [1970].

\section{Spectral Analysis}

Owing to the chemical shift differences induced by the 2 -substituent, the spectrum of the oxetane protons, whose numbering is given in Fig. 1, can

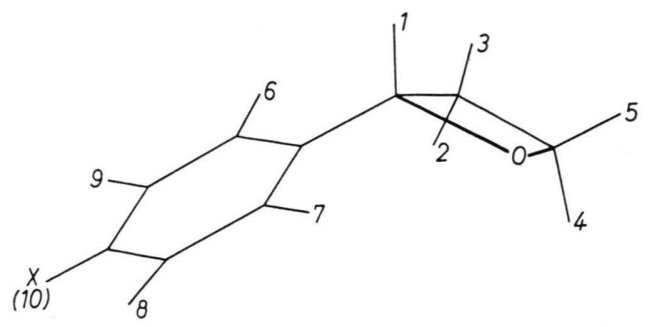

Fig. 1. Numbering used for protons in the 2-(4-halophenyl) oxetanes.

be treated as an ABCDE spin system. The treatment as a 5 -spin system is possible, as the couplings of the protons with the substituent can be ignored. Then the four phenyl protons form an $\mathrm{AA}^{\prime} \mathrm{BB}^{\prime}$ system. When a coupling between the phenyl and oxetane protons exists, the above mentioned grouping in two separate non-interacting $\mathrm{AA}^{\prime} \mathrm{BB}^{\prime}$ and $\mathrm{ABCDE}$ systems is not possible, and the 9-spin system can be analyzed by subspectral division ${ }^{14}$.

The spectra of protons 2,3 and 4, 5 in oxetane, shown in Figs. 2 and 3, were analyzed as the BCDE part of the ABCDE system, without using the lines produced by proton 1 , or the benzyl proton, in the iterative process, because only the triplet composed of three line groups was resolvable (see Fig. 4). The chemical shifts and couplings given by this analysis are collected in Table 1 . The root mean square error, which illustrates the agreement between the spectrum calculated with these parameters and the experimental spectrum, was computed by LAOCN 3 and was $0.049-0.060$, with $62-64$ lines in the fitting.

The phenyl proton spectra of (I) and (II) given in Figs. 5 and 6, and of compound (III) presented in Fig. 7, were analyzed as the $\mathrm{AA}^{\prime} \mathrm{BB}^{\prime}$ and $\mathrm{AA}^{\prime} \mathrm{BB}^{\prime} \mathrm{X}$ parts of $\mathrm{AA}^{\prime} \mathrm{BB}^{\prime} \mathrm{C}$ and $\mathrm{AA}^{\prime} \mathrm{BB}^{\prime} \mathrm{CX}$ spin systems, respectively. The lines of proton 1 , corresponding to part $\mathrm{C}$, were not included in the iterative process. The values of the parameters obtained from the analyses of the phenyl protons are presented in Table 2 .

18 J. Jokisaari and A. Sirkaluoma, Suomen Kemistilehti B 43, 11 [1970].

19 S. Castellano and A. A. Bothner-By, J. Chem. Phys. 41, 3863 [1964]. 


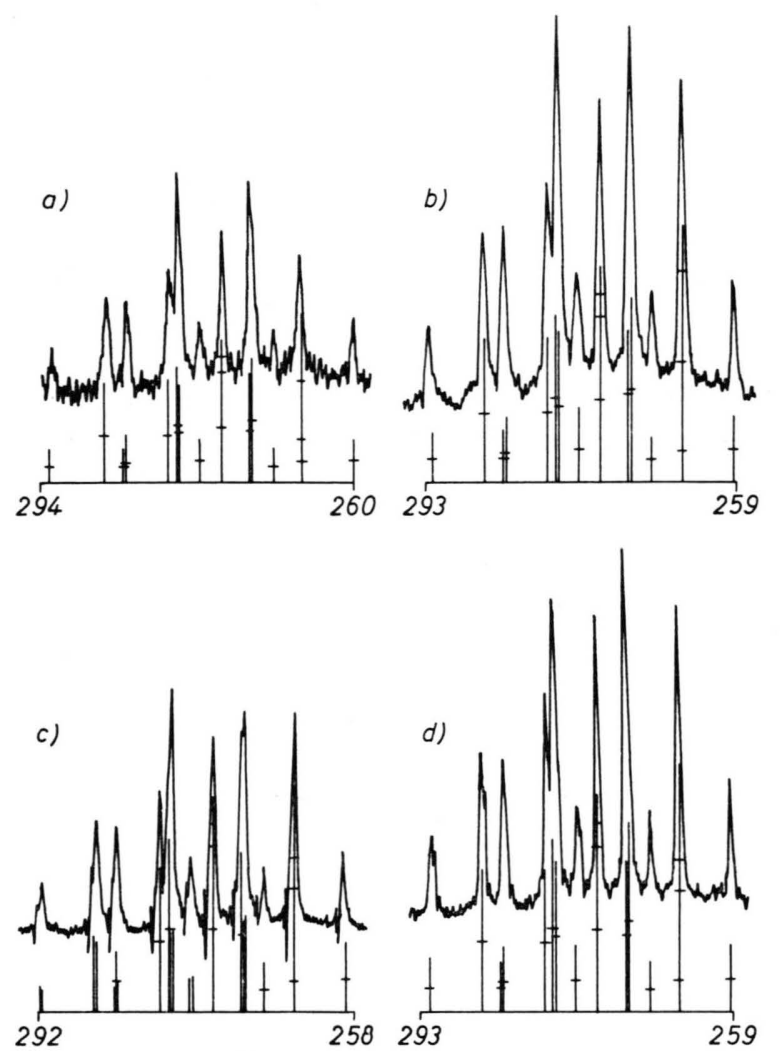

Fig. 2. Observed and calculated spectra of protons 2 and 3 in a) iodo, b) bromo, c) chloro, and d) fluoro derivatives.

a)
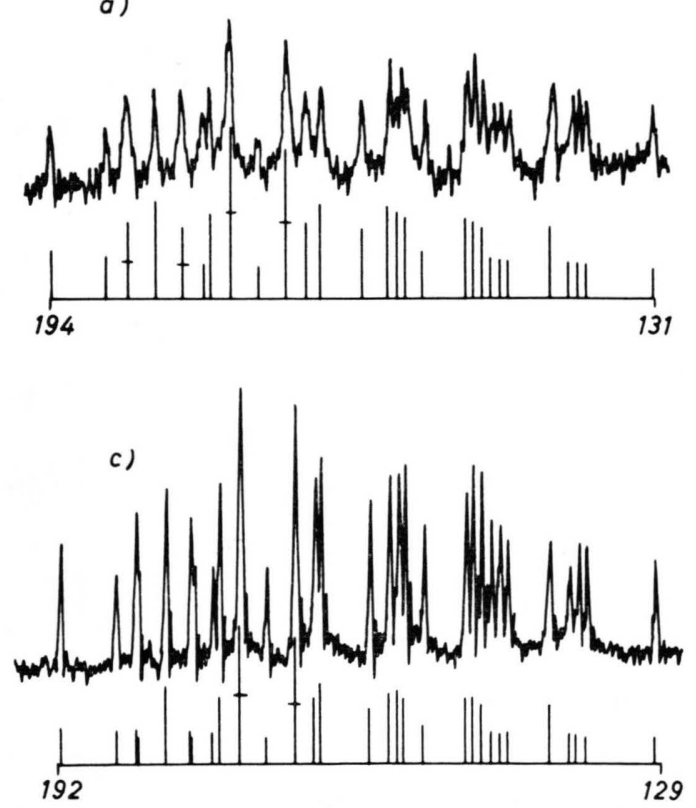

In the iterative analyses of the phenyl protons of (I) and (II) were included 31 and 32 assigned lines, and the rms errors corresponding to the best fittings were 0.072 and 0.068 . In the phenyl proton spectrum of (III) 56 assigned lines produced rms error 0.078. The good agreement between the experimental and theoretical spectra (cf. Figs. 2, 3, $5-7$ ), the latter calculated with the parameters given in Tables 1 and 2, supports the assumption that the couplings with the phenyl protons other than $J_{16}$ and $J_{17}$ have only a slight effect on the structures of the spectra.

Thus it is possible to calculate the triplet produced by proton 1 in compounds (I) - (III) as the A part of an $\mathrm{ABCDD}^{\prime}$ spin system. The protons 1, 2 and 3 form the ABC part, and protons 6 and 7 the $\mathrm{DD}^{\prime}$ part. The values of the chemical shifts and the couplings used in these calculations were taken from Tables 1 and 2. In Fig. 4 are shown the theoretical spectra computed with these parameters.

The respective triplet of 2-(4-chlorophenyl) oxexane (IV) was obtained using parameters in Table 1 as the A part of an $A B C D$ spin system, in which proton 5 corresponds to $\mathrm{D}$. This treatment is due to the fact that there are no couplings between the phenyl and oxetane protons in compound (IV).
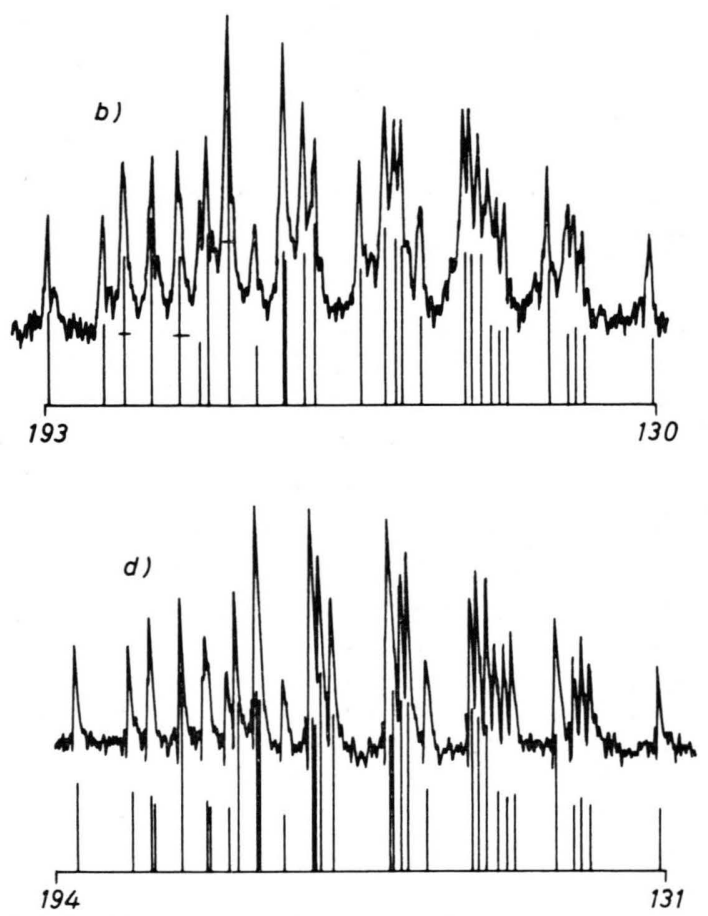

Fig. 3. Observed and calculated spectra of protons 4 and 5 in a) iodo, b) bromo, c) chloro, and d) fluoro derivatives. 

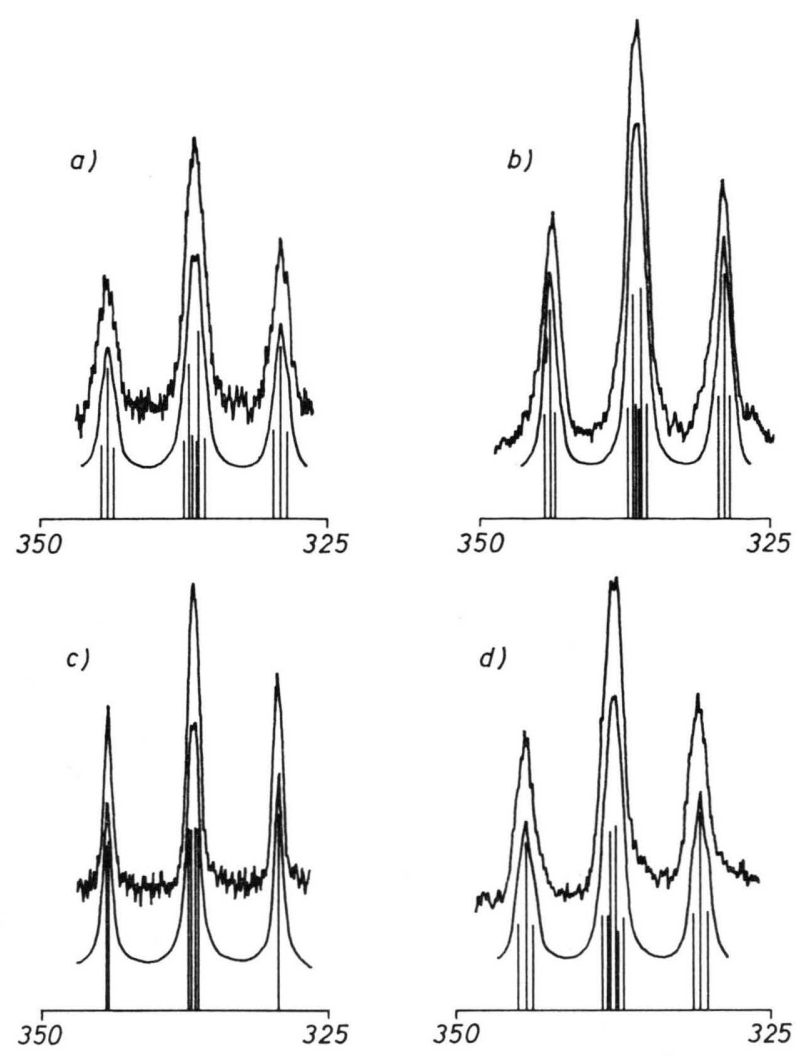

Fig. 4. Triplet of benzyl proton in a) iodo, b) bromo, c) chloro, and d) fluoro derivatives. At the top experimental spectrum and at the bottom calculated line spectrum. Between them triplet computed with $0.4 \mathrm{~Hz}=$ half-width of a single spectral line.

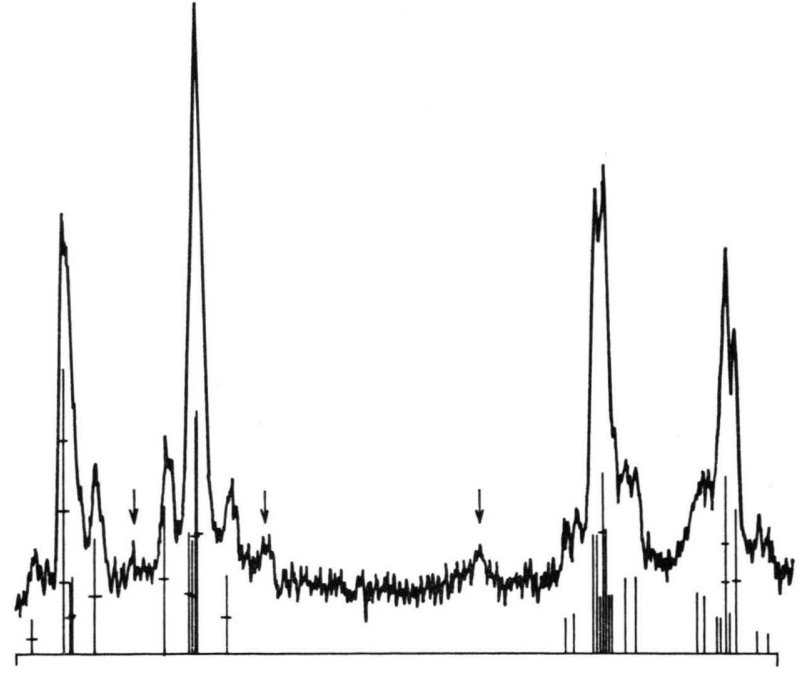

Fig. 5. Observed and calculated spectra of phenyl protons in 2-(4-iodophenyl) oxetane. Resonances marked by arrows are due to impurity.

That is shown by the narrow singlet of the phenyl protons in position $435.66 \mathrm{~Hz}$ relative to TMS (half-width $0.4 \mathrm{~Hz}$ equal to the half-width of the TMS line). Another aim was to include the effect of the coupling $J_{15}=-0.16 \mathrm{~Hz}$ on the calculated spectrum. The value of $0.4 \mathrm{~Hz}$ was used as a halfwidth of a single spectral line in calculating the theoretical shape of the triplet.

Table 1. Chemical shifts a and spin-spin couplings of protons in oxetane ring with their error limits $\mathrm{b}$.

\begin{tabular}{|c|c|c|c|c|c|c|}
\hline & \multicolumn{6}{|c|}{ Substituent $\mathrm{X}$ in phenyl ring } \\
\hline & $\mathrm{F}$ & $\mathrm{Cl}$ & $\mathrm{Br}$ & J & $\mathrm{H}^{\mathrm{c}}$ & $\mathrm{CH}_{3}{ }^{\mathrm{d}}$ \\
\hline$\nu_{1}$ & $338.12 \pm 0.05$ & $336.64 \pm 0.05$ & $336.34 \pm 0.05$ & $336.45 \pm 0.05$ & $347.61 \pm 0.10$ & $335.60 \pm 0.10$ \\
\hline$v_{2}$ & $150.66 \pm 0.02$ & $148.67 \pm 0.02$ & $149.11 \pm 0.01$ & $149.88 \mp 0.02$ & $157.97 \pm 0.05$ & $149.97 \pm 0.05$ \\
\hline$v_{3}$ & $174.94 \pm 0.01$ & $174.84 \pm 0.01$ & $175.91 \pm 0.01$ & $177.05 \pm 0.01$ & $178.73 \pm 0.05$ & $172.00 \pm 0.05$ \\
\hline$v_{4}$ & $269.85+0.02$ & $269.45 \mp 0.02$ & $269.97 \mp 0.02$ & $270.79 \mp 0.01$ & $277.40 \mp 0.05$ & $268.38 \pm 0.05$ \\
\hline$v_{5}$ & $280.30 \pm 0.02$ & $279.85 \pm 0.02$ & $280.39 \pm 0.02$ & $281.21 \mp 0.02$ & $287.36 \pm 0.05$ & $277.95 \pm 0.05$ \\
\hline$J_{12}$ & $7.17 \pm 0.03$ & $7.11 \pm 0.03$ & $7.10 \pm 0.03$ & $7.06 \pm 0.03$ & $7.31 \pm 0.05$ & $7.17 \pm 0.05$ \\
\hline$J_{13}$ & $7.80 \pm 0.02$ & $7.88 \pm 0.03$ & $7.93 \pm 0.02$ & $8.01 \pm 0.03$ & $7.79 \pm 0.05$ & $7.98 \pm 0.05$ \\
\hline$J_{14}$ & $-0.03 \pm 0.02$ & $-0.04 \pm 0.03$ & $-0.02 \pm 0.02$ & $-0.02 \pm 0.03$ & $-0.02 \pm 0.05$ & $-0.03 \pm 0.05$ \\
\hline$J_{15}$ & $0.06 \mp 0.03$ & $-0.16+0.03$ & $-0.07 \mp 0.03$ & $-0.11+0.03$ & $-0.03 \mp 0.05$ & $-0.03 \pm 0.05$ \\
\hline$J_{23}$ & $-10.83 \pm 0.02$ & $-10.84 \pm 0.02$ & $-10.82 \mp 0.01$ & $-10.79 \pm 0.02$ & $-10.91 \mp 0.05$ & $-10.76 \pm 0.05$ \\
\hline$J_{24}$ & $9.13 \mp 0.02$ & $9.11+0.02$ & $9.11+0.02$ & $9.10+0.02$ & $9.15+0.05$ & $9.12+0.05$ \\
\hline$J_{25}$ & $7.85 \pm 0.02$ & $7.82 \pm 0.02$ & $7.85 \pm 0.02$ & $7.78 \pm 0.02$ & $7.74 \pm 0.05$ & $7.75 \pm 0.05$ \\
\hline$J_{34}$ & $5.63+0.02$ & $5.67+0.02$ & $5.70+0.02$ & $5.72+0.02$ & $5.62+0.05$ & $5.62 \pm 0.05$ \\
\hline$J_{35}$ & $8.05 \pm 0.02$ & $8.06 \pm 0.02$ & $8.03 \pm 0.02$ & $8.04 \pm 0.02$ & $8.19 \pm 0.05$ & $8.11 \pm 0.05$ \\
\hline$J_{45}$ & $-5.70 \pm 0.02$ & $-5.75 \pm 0.02$ & $-5.75 \pm 0.02$ & $-5.79 \pm 0.02$ & $-5.75 \pm 0.05$ & $-5.70 \pm 0.05$ \\
\hline
\end{tabular}

a In Hz relative to internal TMS.

b The error limits given for parameters of halogen-substituted 2-phenyloxetanes are probable errors produced by LAOCN 3. Estimated error limits have been used for $v_{1}$ as well as the parameters in the two last columns.

c From Ref. ${ }^{13}$. d From Ref. ${ }^{14}$. 


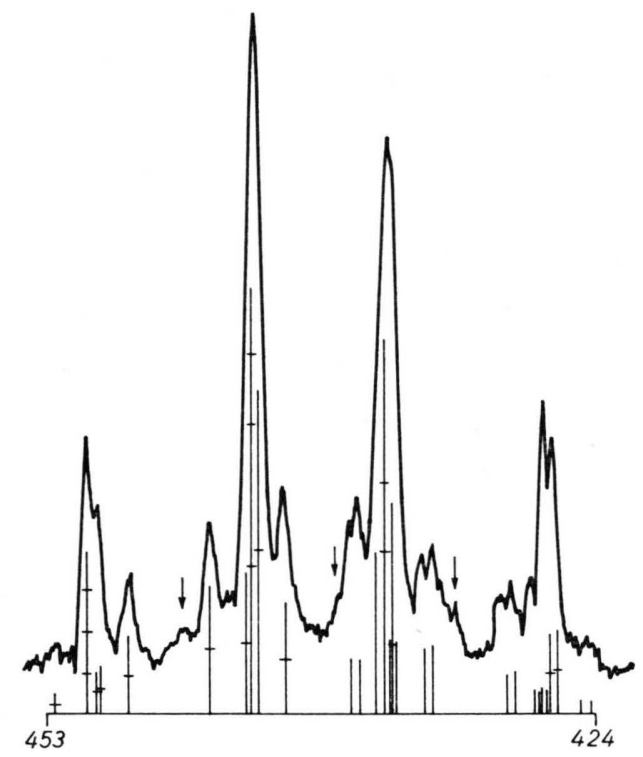

Fig. 6. Observed and calculated spectra of phenyl protons in 2-(4-bromophenyl) oxetane. Resonances marked by arrows are due to impurity.

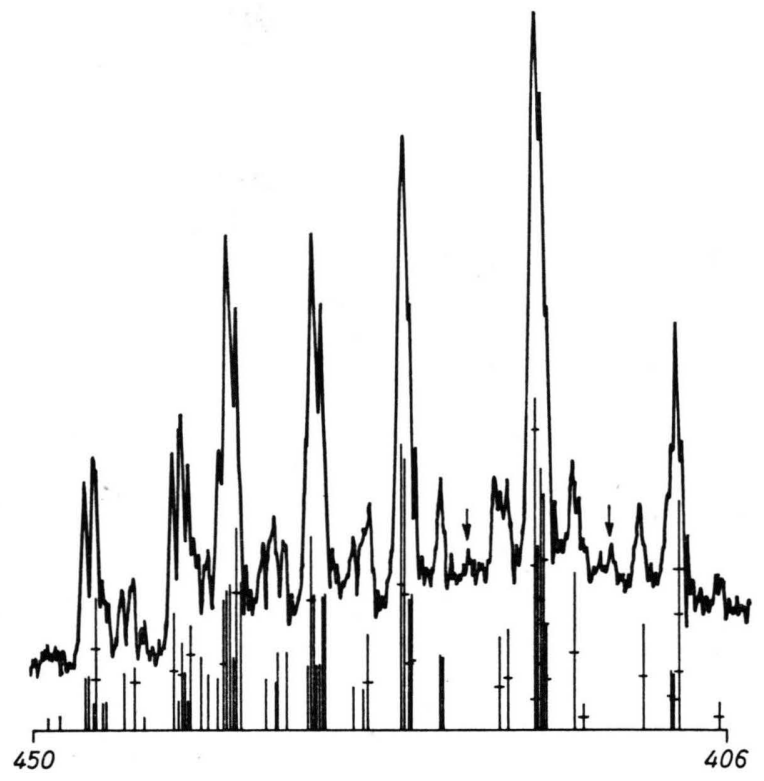

Fig. 7. Observed and calculated spectra of phenyl protons in 2-(4-fluorophenyl) oxetane. Peaks marked by arrows are due to noise.

\section{Discussion}

\section{Oxetane Protons}

The halogen substituent affects primarily the couplings $J_{12}$ and $J_{13}$. The former increases and the latter decreases with growing electronegativity of
Table 2. Proton chemical shifts a, proton-proton and protonfluorine couplings in phenyl ring with their error limits b.

\begin{tabular}{lrrr}
\hline & \multicolumn{3}{c}{ Substituent X in phenyl ring } \\
& $\mathrm{F}$ & $\mathrm{Br}$ & $\mathrm{J}$ \\
\hline$v_{6,7}$ & $438.24 \pm 0.02$ & $431.95 \pm 0.02$ & $424.74 \pm 0.02$ \\
$v_{8,9}$ & $418.64 \pm 0.02$ & $445.20 \pm 0.02$ & $458.30 \pm 0.02$ \\
$J_{68}^{\mathrm{p}}=J_{79}^{\mathrm{p}}$ & $0.30 \pm 0.02$ & $0.41 \pm 0.03$ & $0.42 \pm 0.02$ \\
$J_{89}^{\mathrm{m}}$ & $2.62 \pm 0.02$ & $2.03 \pm 0.03$ & $1.96 \pm 0.04$ \\
$J_{67}^{\mathrm{m}}$ & $2.47 \pm 0.02$ & $2.32 \pm 0.04$ & $2.08 \pm 0.04$ \\
$J_{69}^{\circ}=J_{78}^{\circ}$ & $8.48 \pm 0.03$ & $8.36 \pm 0.03$ & $8.07 \pm 0.03$ \\
$J_{16}=J_{17}$ & $0.63 \pm 0.02$ & $0.46 \pm 0.04$ & $0.57 \pm 0.03$ \\
$J_{\mathrm{HF}}^{\mathrm{o}}$ & $8.64 \pm 0.03$ & - & - \\
$J_{\mathrm{HF}}^{\mathrm{m}}$ & $5.28 \pm 0.03$ & - & - \\
\hline
\end{tabular}

a In $\mathrm{Hz}$ relative to internal TMS.

b Probable errors given by LAOCN 3.

the substituent (see Table 1). However, in 2-phenyloxetane $^{13} J_{12}$ is greater and $J_{13}$ smaller than the respective couplings in 2-(4-iodophenyl) oxetane, although the electronegativity of hydrogen is smaller than that of iodine. On the contrary, with decreasing electronegativity of the substituent, the sum of the trans and cis couplings $J_{12}$ and $J_{13}$, increases:

\begin{tabular}{cccccc}
\hline Sum & F & Cl & Br & I & H \\
\hline$J_{12}+J_{13}$ & 14.97 & 14.99 & 15.03 & 15.07 & $15.10 \mathrm{~Hz}$ \\
\hline
\end{tabular}

The diagonal couplings $J_{14}$ and $J_{15}$ are nearly zero and their error limits are apparently greater than the probable errors produced by LAOCN 3 given in Table 1, since the components of the triplet are not included in the iteration.

The geminal couplings $J_{23}$ and $J_{45}$ are practically independent of the halogen substituent, and their magnitudes correspond to the values found for oxetane ${ }^{3,4}$ and $2,2-\mathrm{d}_{2}$-oxetane ${ }^{5}$. The previous parts of this series have shown that the geminal coupling $J_{23}$ in methyl-substituted 2-phenyloxetanes ${ }^{14}$ and in 2methyloxetane $^{15}$ varies between $-10.42 \mathrm{~Hz}$ and $-10.77 \mathrm{~Hz}$. Instead, the geminal coupling $J_{45}$ remains, in the error limits, equal to the corresponding coupling in the halogen substituted 2-phenyloxetanes studied in the present work. Thus the inductive effect of the 2 -substituent is reduced relative to the distance, having no further effect on the coupling between the protons 4 and 5 , nor on their $\mathrm{H}-\mathrm{C}-\mathrm{H}$ angle ${ }^{20}$.

20 A. A. Bothner-By, in: Advances in Magnetic Resonance, Vol. 1, Ed. J. S. WAUGH, Academic Press, New York 1965, p. 195. 
The vicinal cis couplings $\left(J_{24}\right.$ and $\left.J_{35}\right)$ and the trans couplings $\left(J_{25}\right.$ and $\left.J_{34}\right)$ are practically equal in all the compounds presented in Table 1 .

As to the chemical shifts, the most prominent changes appear in $v_{1}$. That can be interpreted to be partly due to the differences of the electron density of the benzyl carbon between the substitutions, and partly to the stronger effect of the differences in the anisotropy of the magnetic susceptibility of the phenyl ring, caused by the substitution, on the benzyl proton than on the other oxetane protons.

We can state that in all the 2-(4-halophenyl) oxetanes the oxetane protons resonate at a greater value of the applied magnetic field than the corresponding protons in 2-phenyloxetane and, excluding proton 2 , at a lower field than the corresponding protons in 2-(4-methylphenyl) oxetane. It is probable that the shieldings of the protons in the oxetane ring in the different halogen substitutions are principally due to the changes in the ring current, caused by halogens. The magnetic anisotropy of the halogen substituent seems to be a minor factor ${ }^{21}$.

\section{Phenyl Protons}

The phenyl proton couplings in Table 2 are in good agreement with the ranges obtained by previous investigations ${ }^{20,} 22-24$ :

$$
J^{0}=6-8 \mathrm{~Hz}, \quad J^{\mathrm{m}}=1-3 \mathrm{~Hz} \quad \text { and } \quad J^{\mathrm{p}}<1 \mathrm{~Hz} .
$$

The results (cf. Table 2) show that the ortho and meta couplings between the protons in the phenyl ring increase and the para coupling decreases with the growing electronegativity of the substituent in the order: iodine, bromine, fluorine. The meta coupling $J_{89}^{\mathrm{m}}>J_{67}^{\mathrm{m}}$ in the fluoro derivative (III) and $J_{89}^{\mathrm{m}}<J_{67}^{\mathrm{m}}$ in iodo and bromo derivatives (I) - (II).

CASTEllano et al. ${ }^{25,}{ }^{26}$ have stated that in monosubstituted benzenes and in $\mathrm{N}$-substituted pyridines the ortho coupling of the substituent-sided protons and the meta coupling between them increases and the para coupling decreases with the growing elec-

\footnotetext{
21 More information concerning the effect of anisotropy may be obtained after analyzing the spectra of 2-(3,4-dichlorophenyl) oxetane and 2-(2,4-dichlorophenyl) oxetane (under investigation in this lab.).

22 P. Diehl, Helv. Chim. Acta 44, 829 [1961].

23 G. W. Sмith, J. Mol. Spectrosc. 12, 146 [1964].
}

tronegativity of the substituent. But as for the coupling in the same compounds corresponding to the coupling $J_{67}^{\mathrm{m}}$ in phenyloxetanes, they have found that it presents a less definite and more scattered behavior.

If the electronegativity of the oxetane group is assumed to correspond to that of chlorine, as the PMR spectrum of 2-(4-chlorophenyl) oxetane seems to imply, the one of the two meta couplings $J_{67}^{\mathrm{m}}$ and $J_{89}^{\mathrm{m}}$ between the protons which are on the side of the more electronegative para substituent is greater.

Table 3. Chemical shift differences a $\Delta \mathrm{XOx}=v_{6,7}-v_{8,9}$ of phenyl protons in 2-(4-halophenyl)oxetanes, and chemical shift differences a $\triangle \mathrm{XY}$ between protons on the side of substituents $\mathrm{X}$ and $\mathrm{Y}$ measured by DIEHL ${ }^{22}$ in para-dihalosubstituted benzenes ( $\mathrm{a}$ In ppm, $\mathrm{b}=-\Delta \mathrm{FCl}$ ).

\begin{tabular}{llllll}
\hline$\Delta \mathrm{FOx}^{\mathrm{C}}$ & +0.33 & $\Delta \mathrm{BrOx}$ & -0.22 & $\Delta \mathrm{IOx}$ & -0.56 \\
$\Delta \mathrm{ClF}^{\mathrm{b}}$ & -0.32 & $\Delta \mathrm{BrCl}$ & -0.22 & $\Delta \mathrm{ICl}$ & -0.53 \\
\hline
\end{tabular}

The chemical shift equivalence of the phenyl protons in the chloro derivative proves that the effects of chlorine and the oxetane group on the shieldings of the phenyl protons are equal. This observation is supported by the chemical shift differences $\Delta^{\mathrm{X} O \mathrm{x}}$ between the halogen- and oxetane-sided phenyl protons in compounds (I) - (III). These are collected in Table 3 . These differences are practically equal to the ones measured by DIEHL ${ }^{22}$ in para-dihalosubstituted benzenes, in which chlorine is in the same position as oxetane in the compounds that were investigated in the present work.

\section{Acknowledgements}

The authors wish to thank Prof. P. TUомікоsкi for helpful discussions on the manuscript and Mr. E. PäÄKKÖNEN for assistance in the English text. Miss M.-L. Perkkiö, Miss A. Harvala, Mr. Y. Kurth, and Mr. M. LAURONEN are acknowledged for skilful help in the measurements. The investigation was financially supported by the Finnish National Research Council for Sciences.

24 K. Hayamizu and O. Yamamoto, J. Mol. Spectrosc. 25, 422 [1968].

25 S. Castellano and C. Sun, J. Amer. Chem. Soc. 88, 4741 [1966].

26 S. Castellano and R. Kostelnik, J. Amer. Chem. Soc. 90, 141 [1968]. 